\title{
Wigner localization and whispering gallery modes of electrons in quantum dots
}

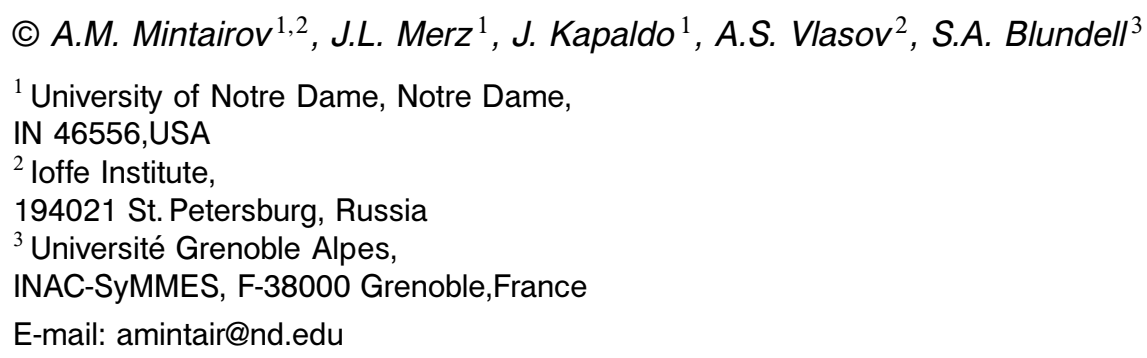

We used a low temperature near-field scanning optical microscopy (NSOM) to study a formation of Wigner molecules (WMs) in the emission spectra of self-organized InP/GaInP QDs having up to seven electrons. We used a Schottky diode structure for the electrostatic control of the number of the electrons $(\mathrm{N})$ in QD, and we observed the emission of the charged excitons (for $N<3$ ) and of the WMs (for $N>2$ ) in the regime of weak Wigner localization. We show, that a contribution of whispering gallery modes (WGMs) of the electrons and rearrangement of the electrons between the WM and WGM states in the QDs, can explain the anomalous dependence of NSOM image size on the quantum confinement observed for some dots.

\section{Acknowledgment}

The work at UND is supported by the National Science Foundation (Grant No ECCS \# 1509087). 